\title{
Progression from impaired fasting glucose and impaired glucose tolerance to diabetes in a high-risk screening programme in general practice: the ADDITION Study, Denmark
}

\author{
S. S. Rasmussen • C. Glümer • A. Sandbaek • \\ T. Lauritzen $\cdot K$. Borch-Johnsen
}

Received: 30 June 2006 / Accepted: 10 October 2006 / Published online: 2 December 2006

(C) Springer-Verlag 2006

\begin{abstract}
Aims/hypothesis To estimate the 1-year progression rates from both IFG and IGT to diabetes in individuals identified in a pragmatic diabetes screening programme in general practice (the ADDITION Study, Denmark [Anglo-DanishDutch Study of Intensive Treatment in People with ScreenDetected Diabetes in Primary Care]).

Methods Persons aged 40-69 years were screened for type 2 diabetes based on a high-risk, stepwise strategy. At baseline, anthropometric measurements, blood samples and questionnaire data were collected. A total of 1,160 persons had IFG or IGT at baseline: $811(70 \%)$ accepted reexamination after 1 year. Glucose tolerance classification was based on the 1999 WHO definition. At follow-up, diabetes was based on one diabetic glucose value of fasting blood glucose or 2-h blood glucose.

Results At baseline, 308 persons had IFG and 503 had IGT. The incidence of diabetes was 17.6 and 18.8 per 100 person-years in the two groups, respectively.

Conclusions/interpretation IFG and IGT identified in general practice during a stepwise, high-risk screening programme for type 2 diabetes have high 1-year progression rates to diabetes. Consequently, intensive follow-up and intervention strategies are recommended for these highrisk individuals.
\end{abstract}

S. S. Rasmussen $(\bowtie) \cdot$ C. Glümer $\cdot$ K. Borch-Johnsen

Steno Diabetes Center,

Niels Steensensvej 2,

DK-2820 Gentofte, Denmark

e-mail: SSSR@steno.dk

\section{A. Sandbaek · T. Lauritzen $\cdot$ K. Borch-Johnsen}

Institution of Public Health, Department of General Practice,

University of Aarhus,

Aarhus, Denmark
Keywords Blood glucose · Denmark · Follow-up study General practice $\cdot$ Impaired fasting glucose . Impaired glucose tolerance $\cdot$ Progression rate . Screening · Type 2 diabetes

\author{
Abbreviations \\ 2hBG 2-h blood glucose \\ ADDITION Anglo-Danish-Dutch Study of Intensive \\ Treatment in People with Screen-Detected \\ Diabetes in Primary Care \\ FBG fasting blood glucose \\ RBG random blood glucose
}

\section{Introduction}

Screening is normally thought of as general populationbased screening, where everybody within a given population and age group is offered a screening test. In everyday clinical work, usually high-risk screening/opportunistic case finding takes place where individuals with several risk factors for having diabetes are being tested. In both strategies, individuals with IFG and IGT are also identified, but their risk of developing diabetes differs according to identification strategy. As the risk reported in the existing literature generally is based on population-based studies and clinical prevention trials, the true risk for individuals identified within high-risk screening strategies is unknown.

Information on the risk of progression in such high-risk screened individuals is highly relevant as means are available to prevent or delay the onset of diabetes [1-5]. Valid estimates of the risk are important to doctors and patients in the assessment of whether they find that the risk and risk reduction justify the necessary lifestyle modifications. 
Our aim was therefore to estimate the 1-year progression rates from IFG and IGT to diabetes in a population-based, high-risk screening programme for type 2 diabetes in general practice.

\section{Subjects and methods}

Study design and population The study population was based on the ADDITION Study, Denmark (Anglo-DanishDutch Study of Intensive Treatment in People with ScreenDetected Diabetes in Primary Care), which is a populationbased, high-risk screening and intervention study for type 2 diabetes in general practice [6]. Evaluation of the screening programme, including a pilot study, has been published elsewhere [7].

Screening for diabetes was conducted in five counties. All persons aged 40-69 years, registered with the participating practices, received an invitation letter with a risk score. The risk score for diabetes was developed in a general population-based screening study in Denmark (the Inter99 study) and validated in the pilot study for the ADDITION study [7,8]. Patients with a risk score of 5 points or more (Fig. 1) were recommended to contact their general practitioner for further tests. In this second step, random blood glucose (RBG) and $\mathrm{HbA}_{1 c}$ were measured. Individuals were considered at low risk of having diabetes if RBG was $<5.5 \mathrm{mmol} / 1$ and $\mathrm{HbA}_{1 c}<5.8 \%$. Cut-points for RBG and $\mathrm{HbA}_{1 c}$ were also based on the pilot study with a sensitivity of $85.7 \%$, a specificity of $75.7 \%$ and a testpositive fraction of $27.7 \%$ when used combined [7]. In all those who proceeded in the programme after this second step, fasting blood glucose (FBG) was measured. An OGTT was performed within the same consultation if FBG was 5.6-6.1 mmol/1 or $\mathrm{HbA}_{1 c} \geq 5.8 \%$. Each step divided persons into a high vs low risk of having diabetes. Those at low risk did not continue in the programme and therefore follow-up data are not available on low-risk individuals.

After 1 year, all persons with elevated, non-diabetic blood glucose levels at baseline were invited to make an appointment with their general practitioner following the same procedure as at baseline, where FBG was measured in all individuals and 2-h blood glucose (2hBG) after an oral glucose load depending on elevated levels of FBG or $\mathrm{HbA}_{1 c}$. The result of $\mathrm{HbA}_{1 c}$ was not given immediately and therefore in cases where $\mathrm{HbA}_{1 c}$ but not FBG was elevated a new appointment was set for the OGTT.

From April 2001 to August 2003, 102,277 persons were addressed, 19,013 persons with a score $\geq 5$ responded and 18,222 of those attended the second screening step, in which 8,060 had elevated $\mathrm{RBG}$ or $\mathrm{HbA}_{1 c}$. Of those, 6,758 persons attended the third step (FBG/2hBG), 1,001 had one

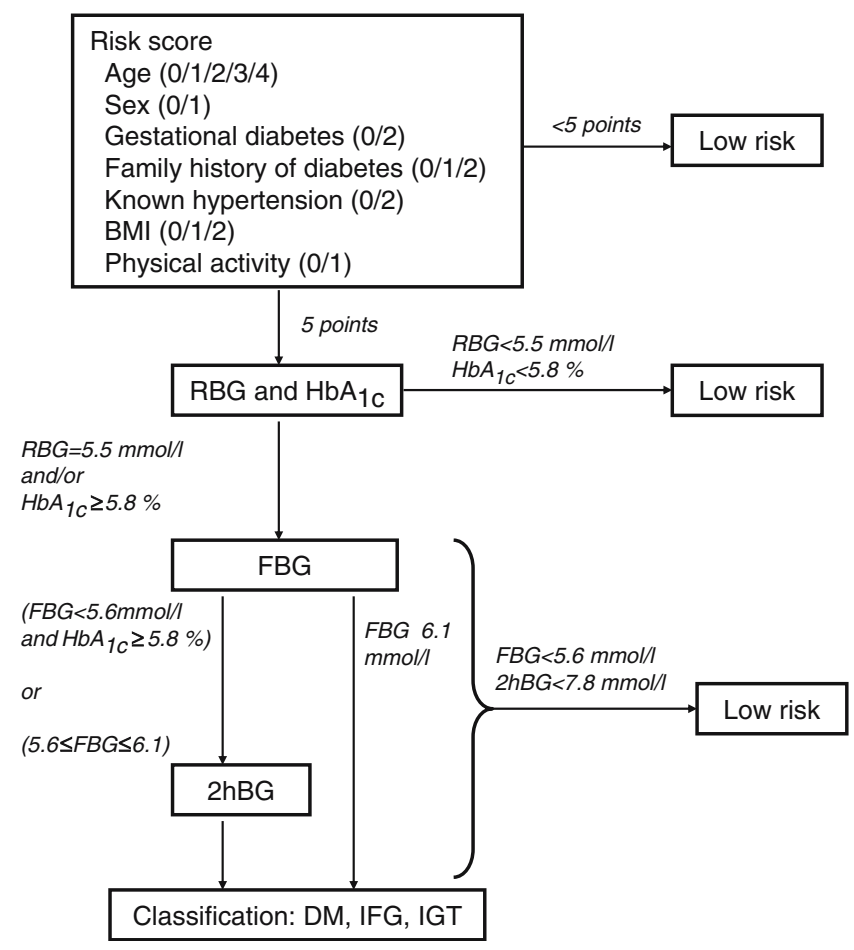

Fig. 1 The screening algorithm in the ADDITION study, Denmark. $D M$, diabetes mellitus

diabetic glucose value and 1,218 were identified with IFG or IGT. Of these 1,218 persons, 58 had missing $2 \mathrm{hBG}$ values, leaving 1,160 for the present follow-up study.

The study was in accordance with the Declaration of Helsinki revised in 1996 and approved by the Scientific Ethics Committee of Aarhus, Denmark. All participants gave written informed consent.

Measurements and definitions As the ADDITION study is based on a clinical approach in general practice, blood glucose was measured on capillary whole blood using a HemoCue B-glucose analyser based on the glucose dehydrogenase reaction (HemoCue AB, Ängelholm, Sweden). All practices were supervised by the HemoCue Company in analysing the blood samples and the daily calibration of the machines [7]. FBG was taken after an overnight fast, where the persons were instructed not to eat, drink or smoke later than $23 \mathrm{~h}$ the evening before. Subsequently, the 75-g OGTT was performed with the persons remaining at the consultation. After $2 \mathrm{~h}$ the $2 \mathrm{hBG}$ was measured. To minimise measurement error based on variation with HemoCue, two capillary blood samples with 1-min intervals were taken and the average of the two results was used [9].

The glucose tolerance classification was based on FBG and 2hBG using the WHO 1999 definition [10]: IFG ( $5.6 \mathrm{mmol} / 1 \leq \mathrm{FBG}<6.1 \mathrm{mmol} / 1$ and $2 \mathrm{hBG}<7.8 \mathrm{mmol} / \mathrm{l}$ ); IGT $(\mathrm{FBG}<6.1 \mathrm{mmol} / 1$ and $7.8 \mathrm{mmol} / 1 \leq 2 \mathrm{hBG}<$ $11.1 \mathrm{mmol} / \mathrm{l})$ and diabetes $(\mathrm{FBG} \geq 6.1 \mathrm{mmol} / 1$ or $2 \mathrm{hBG} \geq$ 
$11.1 \mathrm{mmol} / \mathrm{l})$. At follow-up, incident diabetes was defined as one diabetic glucose value of FBG or $2 \mathrm{hBG}$. In addition, confirmation of diabetes with two diabetic values of FBG or $2 \mathrm{hBG}$ on two separate days was advised to identify cases of clinical diabetes.

Fasting venous blood samples were mailed together with a spot urine to the central laboratory (University Hospital of Aarhus). $\mathrm{HbA}_{1 c}$ was analysed using liquid chromatography on a Tosoh machine (TOSOH Alc 2.2; TOSOH/Eurogenetics, Germany; normal range 4.2-6.3\%). The Hitachi 971 system (Roche Diagnostics GmbH, Mannheim, Germany) was used to measure total serum cholesterol, serum HDL and serum triacylglycerol by enzymatic tests, urinary albumin by an immunoturbidimetric method and urinary creatinine by a colorimetric method. Serum LDL was calculated using Friedewald's formula. Microalbuminuria was defined as a urinary albumin:creatinine ratio between 2.0 and $25 \mathrm{mg} / \mathrm{mmol}$ and macroalbuminuria as a urinary albumin:creatinine ratio $>25 \mathrm{mg} / \mathrm{mmol}$.

Blood pressure was measured while sitting after a 10 -min rest with a standard sphygmomanometer in each practice. The lowest value of two measurements was recorded. Height and weight were measured wearing light clothing and no shoes, and BMI was calculated as weight divided by the square of the height $\left(\mathrm{kg} / \mathrm{m}^{2}\right)$. Waist circumference was measured midway between the lowest rib and the iliac crest on standing persons. Two selfadministered questionnaires were used: the risk score and a baseline questionnaire on medical history, family history of diabetes, and lifestyle. Information on medication was not given, and information on death among participants was drawn from central registers.

Statistical analyses Baseline data are means \pm SD. Continuous variables were compared by the two-sided $t$ test, categorical variables by Pearson's chi-square. To enable the comparison with other studies, serum triacylglycerol is also given as mean $\pm \mathrm{SD}$, although it was not normally distributed. A $p<0.05$ was considered significant.

Progression rates were estimated by dividing the number of diabetes cases by person-years. Entry date was the date where the first OGTT was done and exit date was the date of the follow-up visit.

\section{Results}

Of the 1,160 individuals identified at baseline there were follow-up data for 811 (70\%) after 1 year (IFG: $n=308$ and IGT: $n=503)$. Five of the 1,160 persons died and for six persons the general practitioner dropped out of the study. The mean follow-up time was 12.5 months with 155 new cases of diabetes ( 38 had a diabetic level on the FBG and
115 had a diabetic level on the $2 \mathrm{hBG}$, while two cases had both diabetic FBG and $2 \mathrm{hBG}$ ). The progression rate for IFG was 17.6 and for IGT 18.8 cases per 100 person-years (Fig. 2). Confirmatory testing was done in 136 of the 155 incident diabetes cases and diabetes was confirmed in $62 \%$ of these cases.

When analysing IGT as two separate categories, i.e. isolated IGT and combined IFG + IGT, 296 had isolated IGT (35 developed diabetes) and 207 had combined IFG + IGT (60 developed diabetes). The progression rates were 12.0 and 28.1 diabetes cases per 100 person-years $(p=0.01)$, respectively (Fig. 2).

Attendees at the follow-up visit did not differ significantly from non-attendees regarding most baseline factors: age, sex, family history of diabetes, BMI, and levels of $\mathrm{HbA}_{1 c}$, FBG, total serum cholesterol, serum LDL and serum triacylglycerol, and systolic BP. However, nonattendees had lower $2 \mathrm{hBG}$ than attendees $(7.7 \mathrm{vs}$ $8.0 \mathrm{mmol} / 1, p=0.01)$. There was a tendency towards lower follow-up rates for IFG than for IGT but the difference was not statistically significant (67.3 vs $71.8 \%, p=0.10)$.

Significantly more individuals with IGT than with IFG had a history of myocardial infarction, 14\% had microalbuminuria and their systolic BP was higher (Table 1).

\section{Discussion}

To the best of our knowledge, we are the first to report on progression rates from IFG and IGT to diabetes in a pragmatic, high-risk screening programme for type 2 diabetes in general practice. Normally, screening strategies are based on progression rates from general populationbased studies referred to in textbooks and guidelines. Our study confirms that these are too low for high-risk settings, which would be the preferable strategy as general popula-

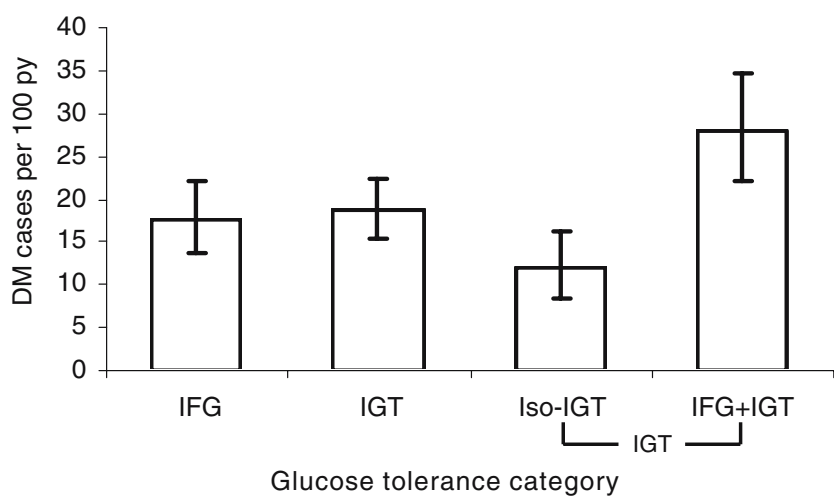

Fig. 2 Progression rates to diabetes in the ADDITION study, Denmark. $D M$, diabetes mellitus; $p y$, person years $(95 \% \mathrm{CI})$. The glucose tolerance categories are defined by WHO 1999 as IFG and IGT. The IGT group is additionally separated into isolated-IGT (Iso-IGT) and combined IFG + IGT 
Table 1 Study characteristics of individuals with IFG and IGT in the ADDITION study. Data are means \pm SD or percentages

\begin{tabular}{llll}
\hline Characteristic & IFG & IGT & $p$ value $^{\mathrm{a}}$ \\
\hline $\begin{array}{l}\text { Number of participants } \\
\text { Progression rate }\end{array}$ & 308 & 503 & \\
$\quad$ (\% per person-year) & 17.6 & 18.8 & \\
Follow-up time (years) & $1.1 \pm 0.4$ & $1.0 \pm 0.5$ & \\
Age (years) & $59.8 \pm 6.4$ & $61.3 \pm 6.6$ & 0.002 \\
Sex (\% women) & 39.9 & 55.1 & $<0.001$ \\
BMI (kg/m ${ }^{2}$ ) & $28.9 \pm 4.4$ & $29.5 \pm 5.2$ & 0.07 \\
Waist (cm) & $97.9 \pm 11.5$ & $98.4 \pm 14.0$ & 0.63 \\
WHR & $0.92 \pm 0.09$ & $0.90 \pm 0.09$ & 0.02 \\
HbA $1 c$ (mean \%) & $5.6 \pm 0.4$ & $5.9 \pm 0.4$ & $<0.001$ \\
FBG (mmol/1) & $5.8 \pm 0.1$ & $5.3 \pm 0.5$ & $<0.001$ \\
2hBG (mmol/1) & $6.2 \pm 1.1$ & $9.1 \pm 0.9$ & $<0.001$ \\
Systolic BP (mmHg) & $140 \pm 17$ & $143 \pm 19$ & 0.03 \\
Total cholesterol (mmol/l) & $5.7 \pm 1.0$ & $5.8 \pm 1.1$ & 0.05 \\
HDL (mmol/l) & $1.6 \pm 0.4$ & $1.5 \pm 0.4$ & $<0.001$ \\
LDL (mmol/1) & $3.4 \pm 0.8$ & $3.6 \pm 1.0$ & 0.004 \\
Triacylglycerol (mmol/1) & $1.4 \pm 0.8$ & $1.6 \pm 0.9$ & 0.001 \\
Microalbuminuria (\%) & 7.7 & 14.7 & 0.008 \\
Family history & 32.2 & 35.6 & 0.34 \\
of diabetes (\%) $^{\mathrm{b}}$ & & & \\
History of myocardial & 1.3 & 5.7 & 0.001 \\
infarction (\%) & & & \\
\hline
\end{tabular}

${ }^{\mathrm{a}} p$ value for comparing IFG and IGT

${ }^{\mathrm{b}}$ Because of missing data, denominators vary for microalbuminuria (IFG $n=274$, IGT $n=426$ ), family history of diabetes (IFG $n=292$, IGT $n=481$ ) and history of myocardial infarction (IFG $n=300$, IGT $n=487$ )

tion-based screening programmes are expensive [11], logistically difficult and of questionable benefit $[12,13]$.

Healthcare providers and high-risk individuals naturally meet in general practice. Inviting patients via their general practitioners is a population-based approach in Denmark, as $97 \%$ of the population are registered in a general practice. Because the ADDITION study is pragmatically evaluating a screening strategy designed for future national implementation, it is based on patient's self-referral after receiving the risk score and the follow-up invitation. This screening strategy has been evaluated elsewhere [7] and the present study concentrates on the follow-up of individuals with IFG and IGT.

The 1-year follow-up rate was $70 \%$. This is in accord with that found in the observational Hoorn study [14], and for conditions not disease labelled [15] a higher attendance rate may be unrealistic. It is possible that only those with multiple risk factors, and thus higher risk, attended for follow-up, causing an overestimation of progression rates. The IFG group tended to have a lower follow-up rate than the IGT group, who had a worse cardiovascular disease risk profile. At follow-up, non-attendees of the entire population did not differ from attendees by major risk factors for progression, although a difference too small to explain the high progression rates in our study was seen in the mean 2hBG.

Analyses of plasma glucose would require immediate centrifuging and cooling of the blood samples and posting to the laboratory, conditions which we were unable to control. Supported by our pilot study showing similar total analytical variance for blood glucose and plasma glucose [9], we therefore chose duplicate instant measurements of capillary whole blood. Whether individuals are classified identically using different specimens is questionable. The WHO definition of equivalence values for plasma and capillary whole blood may give rise to misclassifications in glucose tolerance status [16].

As risk of diabetes differs between ethnicities [17], we compared our results with studies in primarily whites. In population-based observational studies the annual progression rates were $1-5 \%$ for IFG and $3-11 \%$ for IGT $[14,18-$ 20], whereas we found higher progression rates, and similar for IFG and IGT. In control groups of randomised clinical trials the progression rates from IGT were up to $12 \%$ per year [3-5]. The short follow-up time in our study implies that the risk of diabetes is imminent. Subsequently, the rate may decline. The mean follow-up time in the populationbased screening studies was 4.6-11.5 years [14, 18-20] and in the clinical trials it was about 3 years [3-5], which could indicate that the progression rates in our study will diminish after the first year.

Although the clinical trials based their inclusion strategies on risk factors (age $>25$ years and BMI $>24 \mathrm{~kg} / \mathrm{m}^{2}$ [4] or family history of diabetes, age 40-70 years and BMI $\geq$ $\left.25 \mathrm{~kg} / \mathrm{m}^{2}[3,5]\right)$, there were fewer than in our study. The risk profile in the ADDITION study differed from the control groups in these trials by being older, including more men and having a 7-12 mmHg higher systolic BP, but BMI and waist circumference were smaller [4, 5]. Despite our high-risk approach, the IGT group in the Hoorn study (a classic population-based study) was older, had the same BMI and WHR, a higher systolic BP, and a worse lipid profile [21]. Classic population-based studies are, like our study, based on one OGTT. Some of the clinical trials optimised the identification of high-risk individuals by two repeated OGTTs or restriction to combined IFG + IGT [4, 5], and they repeated the OGTTs 3-monthly or annually $[3,5]$. Nevertheless, the rates in these trials were lower than those in our study, where a higher progression rate for combined IFG + IGT (Fig. 2) was also seen. The difference in progression rates may be better explained by underestimation of the progression rates in the clinical trials, as participants volunteered for the trials and therefore had the potential for lifestyle changes even in the control groups.

In order to compare our data with classic observational studies, we defined diabetes at follow-up by one diabetic value of $\mathrm{FBG}$ or $2 \mathrm{hBG}$. Some clinical trials reported on 
confirmed diabetes [4, 5]. In the ADDITION study, diabetes was confirmed in nearly two-thirds of the cases; thus the progression rate from IGT in our study still lies in the upper range found in these trials. Other studies may identify fewer diabetes cases than the ADDITION study as they used definitions based on a single $2 \mathrm{hBG}$ or two repeated $\mathrm{FBG} \geq 6.7 \mathrm{mmol} / 1$ [20],only fasting glucose [18], or clinically diagnosed diabetes according to registers [19].

High-risk strategies differ, but the practical implications of our results are general. Persons identified with IFG and IGT have a high risk of diabetes (15-20\%) after just 1 year. In Denmark, it is recommended that glucose testing should be done annually in IGT [22] whereas no recommendations are given in IFG or on lifestyle interventions in IFG or IGT [23]. Although evidence is lacking that life-style intervention in IFG is equally effective as in IGT in preventing diabetes, these high-risk individuals should also be offered annual re-examinations for diabetes based on an OGTT.

\begin{abstract}
Acknowledgements We thank B. Carstensen for his statistical assistance, the general practitioners and their staff for their sincere dedication to the ADDITION study and the central laboratory of the University Hospital of Aarhus for handling a large number of blood and urine samples. This fully investigator initiated, designed and controlled study received funding from: the Danish Centre for Evaluation and Health Technology Assessment; the Danish Research Foundation for General Practice; the counties of Aarhus, Copenhagen, Ringkoebing, Ribe and South Jutland; the National Board of Health; the Danish Medical Research Council no 22-04-0390; the Danish Diabetes Association; the A. P. Møller Foundation; the Bernhard and Marie Kleins Trust; Novo Nordisk A/S and Novo Nordisk Scandinavia AB; Astra-Zeneca; Pfizer; Servier; GlaxoSmithKline; and HemoCue.
\end{abstract}

Duality of interest The trial was given unrestricted grants from Novo Nordisk A/S, Novo Nordisk Scandinavia AB, Astra-Zeneca, Pfizer, Servier, GlaxoSmithKline and HemoCue. The authors have no duality of interest.

\section{References}

1. Kosaka K, Noda M, Kuzuya T (2005) Prevention of type 2 diabetes by lifestyle intervention: a Japanese trial in IGT males. Diabetes Res Clin Pract 67:152-162

2. Pan XR, Li GW, Hu YH et al (1997) Effects of diet and exercise in preventing NIDDM in people with impaired glucose tolerance. The Da Qing IGT and Diabetes Study. Diabetes Care 20:537-544

3. Chiasson JL, Josse RG, Gomis R et al (2002) Acarbose for prevention of type 2 diabetes mellitus: the STOP-NIDDM randomised trial. Lancet 359:2072-2077

4. Knowler WC, Barrett-Connor E, Fowler SE et al (2002) Reduction in the incidence of type 2 diabetes with lifestyle intervention or metformin. N Engl J Med 346:393-403

5. Tuomilehto J, Lindstrom J, Eriksson JG et al (2001) Prevention of type 2 diabetes mellitus by changes in lifestyle among subjects with impaired glucose tolerance. N Engl J Med 344:1343-1350
6. Lauritzen T, Griffin S, Borch-Johnsen K et al (2000) The ADDITION study: proposed trial of the cost-effectiveness of an intensive multifactorial intervention on morbidity and mortality among people with type 2 diabetes detected by screening. Int $J$ Obes Relat Metab Disord 24(Suppl 3):S6-S11

7. Christensen JO, Sandbaek A, Lauritzen T, Borch-Johnsen K (2004) Population-based stepwise screening for unrecognised type 2 diabetes is ineffective in general practice despite reliable algorithms. Diabetologia 47:1566-1573

8. Glumer C, Carstensen B, Sandbaek A, Lauritzen T, Jorgensen T, Borch-Johnsen K (2004) A Danish diabetes risk score for targeted screening: the Inter99 study. Diabetes Care 27:727-733

9. Sandbaek A, Lauritzen T, Borch-Johnsen K, Mai K, Christiansen JS (2005) The comparison of venous plasma glucose and whole blood capillary glucose in diagnoses of type 2 diabetes: a population-based screening study. Diabet Med 22:1173-1177

10. Definition, diagnosis and classification of diabetes mellitus and its complications (1999) Report of a WHO consultation, part 1: diagnosis and classification of diabetes mellitus. World Health Organization, Geneva

11. Hoerger TJ, Harris R, Hicks KA, Donahue K, Sorensen S, Engelgau M (2004) Screening for type 2 diabetes mellitus: a costeffectiveness analysis. Ann Intern Med 140:689-699

12. Wareham NJ, Griffin SJ (2001) Should we screen for type 2 diabetes? Evaluation against national screening committee criteria. Br Med J 322:986-988

13. Borch-Johnsen K, Lauritzen T, Glumer C, Sandbaek A (2003) Screening for type 2 diabetes-should it be now? Diabet Med 20:175-181

14. de Vegt F, Dekker JM, Jager A et al (2001) Relation of impaired fasting and postload glucose with incident type 2 diabetes in a Dutch population: the Hoorn study. JAMA 285:2109-2113

15. Unwin N, Shaw J, Zimmet P, Alberti KG (2002) Impaired glucose tolerance and impaired fasting glycaemia: the current status on definition and intervention. Diabet Med 19:708-723

16. Colagiuri S, Sandbaek A, Carstensen B et al (2003) Comparability of venous and capillary glucose measurements in blood. Diabet Med 20:953-956

17. Edelstein SL, Knowler WC, Bain RP et al (1997) Predictors of progression from impaired glucose tolerance to NIDDM: an analysis of six prospective studies. Diabetes 46:701-710

18. Vaccaro O, Ruffa G, Imperatore G, Iovino V, Rivellese AA, Riccardi G (1999) Risk of diabetes in the new diagnostic category of impaired fasting glucose: a prospective analysis. Diabetes Care 22:1490-1493

19. Qiao Q, Lindstrom J, Valle TT, Tuomilehto J (2003) Progression to clinically diagnosed and treated diabetes from impaired glucose tolerance and impaired fasting glycaemia. Diabet Med 20:1027-1033

20. Rajala U, Qiao Q, Laakso M, Keinanen-Kiukaanniemi S (2000) Antihypertensive drugs as predictors of type 2 diabetes among subjects with impaired glucose tolerance. Diabetes Res Clin Pract 50:231-239

21. de Vegt F, Dekker JM, Stehouwer CD, Nijpels G, Bouter LM, Heine RJ (1998) The 1997 American Diabetes Association criteria versus the 1985 World Health Organization criteria for the diagnosis of abnormal glucose tolerance: poor agreement in the Hoorn study. Diabetes Care 21:1686-1690

22. Danish College of General Medicine (2004) Type 2 diabetes in general practice. An evidence-based guidance. 6:1-58 (Report in Danish)

23. National Board of Health, Danish Centre for Evaluation and Health Technology Assessment (2005) Type 2 diabetes. Health technology assessment of screening, diagnosis and treatment. Danish health technology assessment 7. Available from http:// www.sst.dk/publ/publ2005/CEMTV/diabetes_uk/diabetes_uk.pd, accessed 5 May 2005 